\title{
Enhanced uplink scheduling algorithm for efficient resource management in IEEE 802.16
}

\author{
Aneel Oad ${ }^{1,2}$, Shamala K Subramaniam ${ }^{1,2^{*}}$ and Zuriati Ahmad Zukarnain ${ }^{1,2}$
}

\begin{abstract}
Worldwide interoperability for Microwave Access (WiMAX) is based on the IEEE 802.16 standard. This technology provides broadband wireless last-mile access in a Metropolitan Area Network (MAN). The IEEE 802.16-2004 standard (i.e. Fixed WiMAX) provides specification for the Medium Access Control (MAC) and Physical (PHY) layers for WiMAX. A critical part of the MAC layer specification is scheduling, which resolves contention for bandwidth and determines the transmission order of users. The algorithms are researched under different mixes of traffic and for various characteristics of the IEEE 802.16 MAC layer. In this research the focus is on the WiMAX uplink traffic scheduling. A scheduling algorithm's task in a multi-class network is also to categorize the users into one of the pre-defined classes. In this research, the algorithms are tailored at enhancing the collective performance of hybrid algorithms in the WiMAX domains which complement the assigned priorities. The spectrum of constraints which have been extracted from the hybrid Earliest Deadline First(EDF) + Weighted Fair Queuing (WFQ) + First in First out (FIFO) EDF+WFQ+FIFO algorithm include the static nature by which priorities are assigned and maintained during the entire duration of a transmission time and the core attributes of absolute dependence on deadline. The second constraint is embedded within the EDF scheduling algorithm and the perseverance of pursuing deadline associated weightages. In this research, the reengineering of the scheduling mechanics governing the EDF algorithms has been pursued. The dominance of the pre-stipulated deadline of the EDF algorithm is indeed acknowledged in the proposed and developed enhanced algorithm. The simulation results indicate that the legacy algorithms are not suitable for the multi-class traffic systems of WiMAX. This is because these algorithms do not explicitly incorporate the WiMAX QoS parameters into their mechanisms and are highly static. Extensive discrete - event simulation experiments have been done for the purpose of performance analysis. The performance metrics used are average throughput, average delay, missed deadline ratio and average queue size utilization ratio. The acquired results have proven that the proposed algorithms have successfully enhanced the static constrained algorithm.
\end{abstract}

Keywords: Scheduling algorithms; Resource management; Traffic classes; QoS guarantees

\section{Introduction}

The high cost incurred in setting up wired networks especially in rural areas has highly encouraged the expansion of wireless networks. The growing demands (i.e. estimated 25 million by end 2011) [1]) of Internet users has motivated and challenged the innovation of wireless technologies and its respective solutions. Each technology has a unique nature in providing solutions to the challenges imposed onto wireless technologies (i.e. high

\footnotetext{
*Correspondence: shamala@fsktm.upm.edu.my

1 Faculty of Computer Science and Information Technology, Universiti Putra Malaysia, 43400 UPM Serdang, Selangor, Malaysia

${ }^{2}$ Sports Academy, Universiti Putra Malaysia, 43400 UPM Serdang, Selangor, Malaysia
}

variability link, multipath and fading effects). The Worldwide Interoperability for Microwave Access (WiMAX) IEEE 802.16 standard is among the most promising and rising technologies. The salient features of this technology includes its large frequency range, last mile wireless access and increased Quality of Service (QoS) support for various types of applications [2,3]. It efficiently caters the metropolitan area (i.e Wireless Metropolitan Area Networks (WirelessMAN)) demands, with high speed data rate and coverage is up to several kilometers (kms). The essential components of WiMAX include a Base Station (BS) and Subscriber Station (SS). The WiMAX BS can provide broadband wireless access up to 30 miles (i.e. $50 \mathrm{kms}$ ) for fixed stations and between 3 to 10 miles ( 5 to $15 \mathrm{kms}$ )

\section{望 Springer}

(c) 2015 Oad et al: licensee Springer. This is an Open Access article distributed under the terms of the Creative Commons Attribution License (http://creativecommons.org/licenses/by/4.0), which permits unrestricted use, distribution, and reproduction in any medium, provided the original work is properly credited. 
for mobile stations. The BS has a maximum data rate of up to $70 \mathrm{Mbps}$ [4]. It has superior features in comparison to the $802.11 \mathrm{a}$ which has a data rate of $54 \mathrm{Mbps}$ and ranges up to several hundred of meters. The Enhanced Data Rates for Global Evolution (EDGE) has a data rate of $384 \mathrm{kbps}$ and a ranges up to a few $\mathrm{kms}$, and finally the Code-Division Multiple Access 2000 (CDMA2000) which rate is $2 \mathrm{Mbps}$ and a ranges up to a few kms.

This research is focused on WiMAX as the underlying platform. The WiMAX architecture has defined the transmission modes as being Point to Point, Point to Multipoint (PMP) and mesh. In this research, the proposed algorithms are applicable to all modes. The WiMAX architecture is represented by two main layers of the Open System Interconnection (OSI) model which are the Physical (PHY) and the Media Access Control (MAC) layer. The WiMAX PHY layers defined air interfaces are WirelessMAN Single-Carrier (WirelessMANSC) PHY or multiple-carrier PHY layer. The WiMAX multiple-carrier PHY layers air interface are defined as Orthogonal Frequency Division Multiplexing (OFDM) for fixed SSs and the Orthogonal Frequency Division Multiple Access (OFDMA) are used for both fixed and mobile SSs. These two PHY layers air interfaces are using the Time Division Multiple Access (TDMA) technique as a sharing mechanism among multiple SSs. The multiple-carrier PHY spectrum works under the 2$11 \mathrm{GHz}$ range for the fixed and mobile stations and has the Line of Sight (LOS) and Non LOS features $[4,5]$. The WiMAX MAC layer is designed and implemented with some advanced features to provide efficiency, flexibility, encryption, error correction, link adaptation, power control, security, Automatic Retransmission Request (ARQ) and QoS for Uplink (UL) and Downlink (DL) traffic [6,7]. The MAC layer comprises of three sub-layers which are the service specific Convergence Sub-layer (CS), the Common Part Sub-layer (CPS) and the Security Sub-layer [8,9]. These layers are illustrated in Figure 1 [9].

Each of these MAC sub-layers are defined with its respective characteristics. The MAC CS layer is the main function to classify and maps external network data through the CS Service Access Point (SAP) and converts these data into the MAC Protocol Data Units (MPDUs) for transmission over the air. The CS layer specifies the two types of traffic which are transported. They are Asynchronous Transfer Mode (ATM) and Internet Protocol (IP) packets. Each of which is identified with a Connection Identifier (CID) [10]. On the other sub layers, the MAC CPS layer is the main part of the MAC layer which defines the medium access method and specifies the functionality of the system access, bandwidth allocation, scheduling, contention mechanism, connection establishment, and connection maintenance [9]. It receives data from

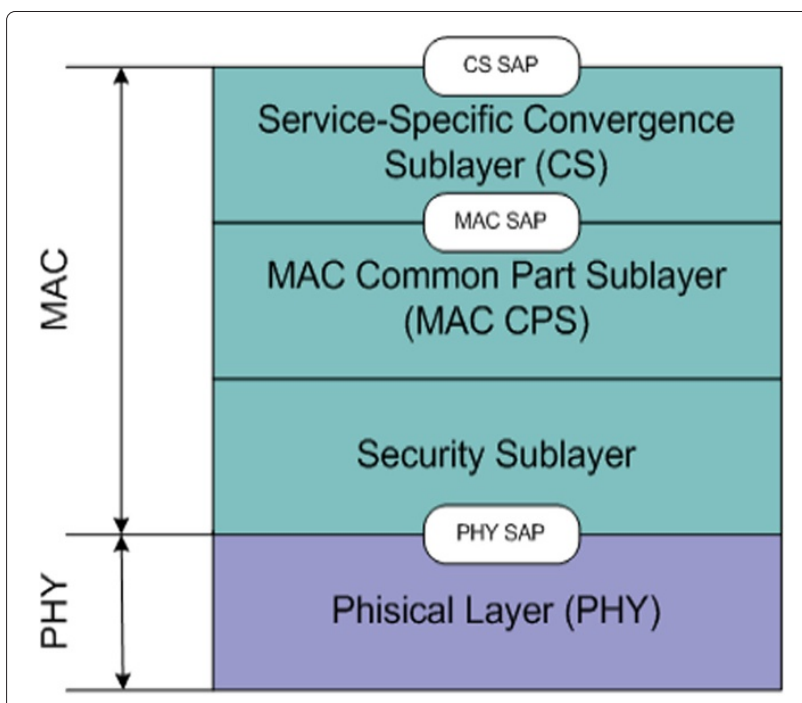

Figure 1 The WiMAX MAC layer [9].

various CSs through the MAC SAP, which is classified to a particular MAC connection [6]. The MAC Security sub-layer lies between the PHY layer and the MAC CPS. The Security sub-layer deals with security issues, authentication and key management. The PHY and MAC layers have constantly received attention and are gradually redesigned to address newly emerging technologies and needs.

The IEEE 802.16e amendment specifies mobile WiMAX that supports both fixed and mobile WiMAX features. However, this standard does not define a MAC scheduling architecture for the UL and DL direction. Thus efficient scheduling design is left to the liberty of the designers. Thus, providing QoS subject to scheduling for IEEE 802.16 BWA system is a challenge for system developers [3]. A scheduling algorithm should take into account the heterogeneous WiMAX QoS classes and service requirements. Scheduling algorithms are used to resolve contention for shared resources in a network. Therefore, it specifies and allocates bandwidth among the users and determines their transmission order to enable improved QoS in WiMAX networks $[3,11,12]$. Several paradigms of scheduling algorithms have been developed to address the evolving nature of WiMAX. Migrating from static schedulers to the hybrid algorithms such as EDF+WFQ+FIFO [13], EDF+WFQ [14], EDF+DFPQ [12] has been among the main fundamental shift.

The rest of this article is organized as follows: Section 2 presents the related works. Section 3 shows the proposed EEF scheduling algorithm. Section 4 presents the performance evaluations of the proposed EEF scheduling algorithm. Section 5 concludes the study, including some ideas for future investigations. 


\section{Related work}

\subsection{The scheduling algorithms}

The scheduling algorithms are used in WiMAX system resolve the contention of bandwidth allocation and to satisfy the QoS among users with different traffic classes. In view of the importance of individualistic characteristics of traffic classes, the expansion of the 802.16-d to harness mobility which resulted in the $802.16 \mathrm{e}$ standard, has impacted the QoS traffic classification. Expanding the four (4) classes of the 802.16-d into five (5) of the 802.16e standard involved the exploitation of the advantages of the UGS and the rtPS traffic. Thus, producing the ertPS traffic [15]. Figure 2 shows the respective classes for each versions of the standard.

In WiMAX networks (IEEE 802.16e, to be precise) of the five QoS classes, three of them are used for real time traffic which are the Unsolicited Grant Services (UGS), Real Time Polling Service (rtPS) and Extended Real Time Polling Service (ertPS). The other two classes used are the non-real time traffic which consist of the Non Real Time Polling Service (nrtPS) and the Best Effort Service (BE) [15]. The UGS class provides a fixed periodic traffic flow with a Constant Bit Rate (CBR) real-time traffic without any form of QoS guarantees. An example of this includes the Voice over Internet Protocol (VoIP) without silence suppression [2,12,14-17]. The rtPS class supports real time traffic flow that generates Variable Bit Rate (VBR) with QoS guarantees in a periodical manner. An example of such traffic are the Motion Picture Expert Group (MPEG), video conferencing and streaming [2,12,14-17]. The nrtPS class is for non-real time VBR traffic with no QoS guarantees (i.e. delay). However, it can provide guarantees in terms of high throughput. The File Transfer Protocol (FTP) application belongs to this class [2,12,14-17]. The BE class has no QoS guarantees and neither guarantees delay nor throughput. This class generates variable sized packet application such as the Hypertext Transport Protocol (HTTP) and the electronic mail (email) $[4,15,17]$. As mentioned earlier the ertPS class in the $802.16 \mathrm{e}$ has been created with the objective of exploiting the advantages of UGS and rtPS class [2]. As opposed to the UGS class, this class is designed to support VoIP traffic with silence suppression and the traffic flow generates variable sized packets. These packets are coupled with QoS guarantees and space which are generated in a periodical manner [2,12,14-17].

The design of scheduling algorithms are challenged by the compulsory need to support different level of services, fairness and implementation complexity [16]. The related work analyzed in this Section has shown that there exist multiple ways to classify the algorithms. Among the classification of UL scheduling algorithms are as follows: i) work-conserving and non-work conserving [18], ii) Homogeneous, Hybrid and Opportunistic [13], and iii) Traditional or contemporary methods developed specially for IEEE 802.16 [19]. The deliberation of each of these methods of classification is done in detail subsection 2.2. The focus of this research is on scheduling algorithms for UL traffic in WiMAX. An uplink scheduling algorithm at the BS has to coordinate its decision with all the SSs whereas the downlink algorithm is only concerned in communicating the decision locally to the BS [12].

\subsection{Classification of uplink WiMAX scheduling algorithms} The IEEE 802.16 has attracted numerous scheduling algorithms with respect to the characteristics of the IEEE 802.16 MAC layer and the OFDM physical layer. These algorithms may either be classified by common advocacies or be developed by a paradigm of design. There are several classification methods deliberated in the literature. One classification method groups algorithms into being either work-conserving or non-work conserving [18]. A work-conserving scheduler is never idle, if there exist a packet in the queue it will be served until all jobs are finished. Whereas in a non-work conserving scheduler, the system can be idle even when there is a packet in the queue waiting for service. A second classification method of scheduling algorithms has classified the algorithms into three categories which are homogenous algorithms, hybrid algorithms and opportunistic algorithms [13]. This classification is based on the essence used for determining the order of packet serving. The homogenous and hybrid categories both consist of legacy algorithms (i.e. legacy algorithms are those inherited from the wired/wireless domain). However, the hybrid category employs multiple legacy schemes which are inter-related/integrated in an

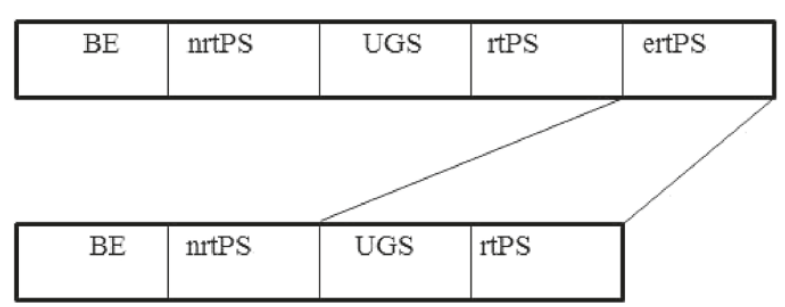

IEEE $802.16 \mathrm{e}$

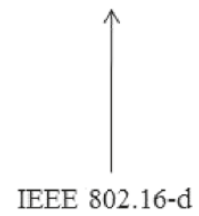

Figure 2 WiMAX traffic classes. 
attempt to satisfy the diverse QoS requirements of the multi-class traffic environment in WiMAX networks. The opportunistic class of scheduling algorithms is proposed for WiMAX to exploit variations in channel quality by giving priority to users with better channel quality, while attempting to satisfy the QoS requirements of multi-class traffic. This class of scheduling algorithms encompasses traditional legacy algorithm and algorithms which have been specially developed for IEEE 802.16 as shown in Figure 3.

Figure 3 is the classification formed based on the varying differentiation and groupings done by the related work. The general approach taken is to customize wired based algorithms such as Round Robin (RR) for WiMAX. The traditional legacy method is based on the adaption of classical algorithms used in wired networks. They have been classified into simple and hierarchical schedulers [19]. The simple traditional scheme is adopted without any modification on its form of the principal architecture when deployed onto the IEEE 802.16. The hierarchical type of mechanisms is proposed specifically for the IEEE 802.16 scheduling. This hierarchical mechanism maintains fairness between the QoS classes and differentiates the services between them. However, the problem with this mechanism is the complexity of implementation. These specifically tailored algorithms developed for the IEEE 802.16 has been further divided into algorithms which are able to be used for all classes of traffic and algorithms which are used for only a specific class of traffic [19]. In the algorithm for all classes of traffic, the proposed solution is capable of allocating slots based on the specific QoS requirements, bandwidth request sizes, and the WiMAX network parameters [20]. However, for the algorithms used for a specific class, it is difficult to estimate the amount of required bandwidth due to the dynamic changes of the sending rate of the traffic. The UL scheduling algorithms classification to the attributes and mechanics of individual algorithms. Each algorithm is unique in its own sense and posses both advantages and disadvantages. Trend analysis of the formation of this research is among the intended outcomes of this discussion.

\subsubsection{The weighted fair queuing algorithm}

The family of Fair Queuing (FQ) algorithms has been among the most prominent scheduling paradigms in origin of it member of the fraternity, the Weighted Fair Queuing (WFQ) algorithm [21]. The WFQ is a packet scheduling scheme that is evaluated to approximate Fluid Fair Queuing (FFQ) or Generalized Processor Sharing (GPS) scheme [22]. The WFQ is also known as Packetized Generalized Processing Sharing (PGPS) that is a packet approximation algorithm for GPS [23]. This discipline was developed independently in 1989 by Lixia Zhang, Alan Demers, Srivinasan Keshav and Scott Shenker. The WFQ supports both, the guaranteed service requirement connection and also connections without guaranteed service requirement [24]. The WFQ assigns a weight to each subscriber the same way as Weighted Round Robin (WRR) and the weight is equal to the amount of a quantum of Deficit Round Robin(DRR) $[25,26]$. However, the argument used to make a scheduling decision is the finish number. The finish number is an estimation of the time at which each individual packet will finish service. The packet with the lowest finish time will be scheduled first. The finish number is calculated based on the subscriber's weight, the finish number of the previous packet scheduled on that connection and the length of the packet [24]. Once the weight is assigned, the arriving

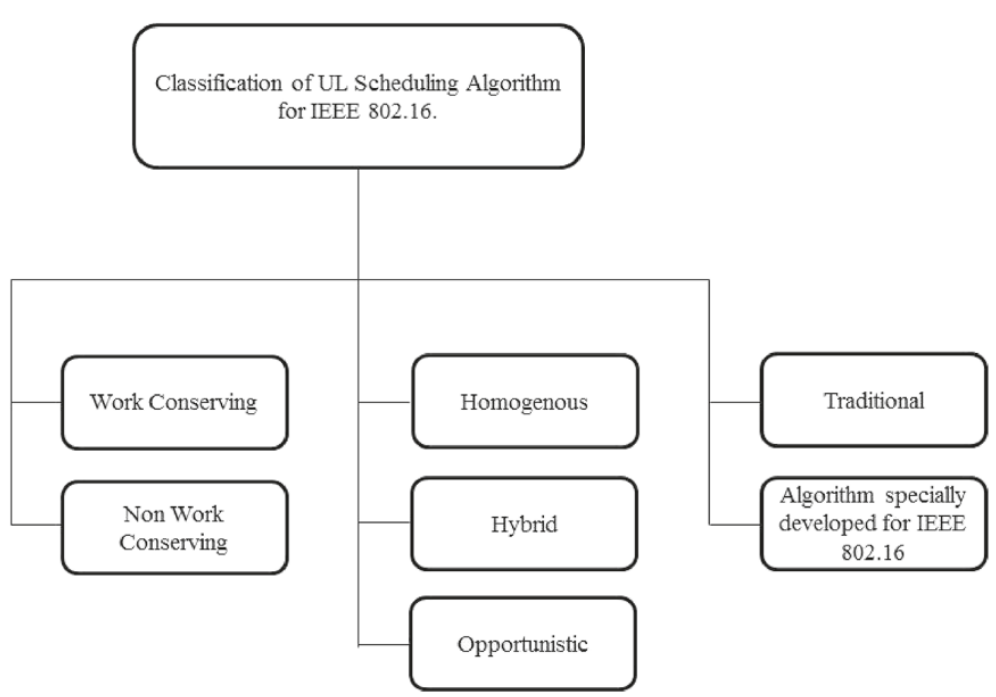

Figure 3 Classification of scheduling algorithms. 
packets of the SS are stamped with a virtual finish time which is calculated using the equation below.

$$
\begin{aligned}
& S_{k}^{i}=\max \left\{F_{i}^{k}-1, V\left(a_{i}^{k}\right)\right\} \\
& F_{i}^{k}=S_{k}^{i}+L_{k}^{i} / \phi_{i},
\end{aligned}
$$

where $S_{k}^{i}$ is the start time of $k$ th packet of $\mathrm{SSi} ; F_{i}^{k}-1$ is the finish time of $(k-1)$ th packet of $\mathrm{SSi} ; V\left(a_{i}^{k}\right)$ is the virtual time of the $k$ th packet of $\mathrm{SSi}$; $a_{i}^{k}$ is the arrival time of $k$ th packet of SSi; $F_{i}^{k}$ is the finish time of $k$ th packet of SSi; $L_{k}^{i}$ is the length of $k$ th packet of $\mathrm{SSi} ; \phi_{i}$ is the reserved rate of $\mathrm{SSi}$, where, $\phi_{i}=C * W i$; and $W i$ is the weight assigned to SSi.

The transmission of a packet will trigger an update to the virtual time of its SS. Subsequently, the virtual time will be updated once a packet has been selected for transmission. For a time interval $\tau$, the virtual time $V(t)$ is calculated as follows:

$$
V\left(t_{(j-1)}+\tau\right)=V\left(t_{j-1}\right)+\frac{\tau}{\sum_{i \in B_{j} \phi_{i}}},
$$

where $\tau \leq t_{j}-t_{j}-1 j=2,3, \ldots$ and $B_{j}$ is the set of busy SSs.

The WFQ does not make the infinitesimal packet size assumption as was done in GPS, and with variable-size packets, they do not need to know a connection's mean packet size in advance [18]. The disadvantage of the WFQ algorithm is that it will serve packets even if they would not have started a service under the GPS algorithm. This is because the WFQ algorithm does not consider the start time of a packet [27]. A tradeoff in the WFQ is its complexity which is high due to the need for selecting the next queue to serve and the computation of the virtual time. The complexity of WFQ is $O(N)$, where $N$ is the number of SSs.

\subsubsection{The earliest deadline first algorithm}

The earliest deadline first (EDF) is a work conserving algorithm which was originally proposed for real-time applications in wide area networks (WAN) [28]. The EDF algorithm as dominant in wired networks has also proven to be useful to WiMAX networks which have been shown in several studies. These results have shown that the EDF successfully achieves minimum delay and low packet loss $[13,17,29,30]$. The EDF algorithm has also been analytically analyzed which have further proven these performance characteristics [31]. The EDF algorithm is a delayoptimal scheduling algorithm at which a single connection in terms of a schedulable region for a set of flows is given with deterministic delay requirements [32]. The EDF algorithm has been declared to be suited for connections with guaranteed service requirement [17]. In this algorithm, each packet is assigned a deadline. The scheduler serves packets in the order of their respective deadlines. The packet with the minimum (i.e., earliest) deadline will be served first. Thus, EDF scheduling tends to give preference to connections with real-time needs. However, it does not provide any form of protection against the dominance of greedy connections, which always set the deadline closer to the arrival time. The server is only able to guarantee that the packet with a smaller deadline will have a higher priority and will be served at the earliest possible time [24]. Table 1 elaborates most capable scheduling algorithms comply in IEEE 802.16.

\subsubsection{Hybrid algorithms}

In deriving various solutions for the optimum UL scheduling algorithm, researches have sorted to combining forces of individual algorithm. These paradigms of design are known as hybrid scheduling. The hybrid scheduling algorithms used in WiMAX networks thus can be considered as a unified algorithm which is formed from a combination of legacy scheduling algorithms to satisfy the QoS requirements of the multi-class traffic as specified in the IEEE 802.16 standard. A number of hybrid scheduling algorithms have been proposed and are discussed in the next section.

One of the pioneering hybrid algorithms is the EDF+WFQ+FIFO scheduling algorithm which was proposed by [13]. It is used in WiMAX networks for managing heterogeneous traffic classes. These classes create an efficient management but impose higher QoS demands in the networks. The hybrid EDF+WFQ+FIFO algorithm service is implemented with different QoS schedulers to meet these requirements. The EDF is used for the rtPS traffic, the WFQ is used for the nrtPS traffic, and the

Table 1 Comparison of legacy algorithms

\begin{tabular}{llllll}
\hline Parameter of comparison & RR & WRR & DRR & WFQ & EDF \\
\hline Complexity & $O(1)$ & $O(1)$ & $O(1)$ & $O(N)$ & $O(N)$ \\
Work conserving & No & Yes & Yes & Yes & Yes \\
Core parameter for scheduling & Time stamped & Weight & Quantum & Weight+ finish number & Deadline \\
Supported traffic & Non-real-time traffic & Non-real time traffic & Non-real time traffic & Real and non-real-time traffic & Real-time traffic \\
\hline
\end{tabular}


FIFO is used for SSs of the BE class [29]. The main reason of motivating this hybrid scheme is the ideal distribution of bandwidth among the diverse traffic classes. There is no single legacy scheduler which can satisfy all the QoS requirements of applications in the WiMAX networks [13]. The hybrid algorithm is formed accordingly to the respective application QoS requirements. If the main objective is to provide low delay and packet loss for the real-time application, the EDF scheduler is selected. However, if the objective is to provide strict fairness between the same classes of traffic, the WFQ is suitable. The hybrid EDF+WFQ+FIFO algorithm uses the strict priority service mechanism (i.e., all the higher priority SSs are allocated with bandwidth until they do not have any packets to send) [29]. A drawback of this hybrid scheme is that lower priority SSs will essentially starve in the presence of a large number of higher priority SSs due to the strict priority of overall bandwidth allocation [12].

Another hybrid algorithm proposed for the UL scheduling in IEEE 802.16 network is the EDF+WFQ [30]. This hybrid algorithm is a combination of the EDF (i.e., SSs of the rtPS class) and the WFQ (i.e., SSs of nrtPS and BE classes). The WFQ and the EDF both require the computation of the virtual start time and finish time at the BS [14]. This algorithm differs from the previously explained algorithm in the role of WFQ which caters both the nrtPS and the BE as opposed to only the nrtPS. Thus, the WFQ is used for SSs of both the nrtPS and the BE classes, and the overall bandwidth is allocated fairly. However, the authors have used a computationally complex algorithm (i.e., WFQ is used for the class of BE traffic which do not required any QoS guarantees) [12,27].

Another hybrid scheduling algorithm is the combination of EDF+WFQ which was proposed by [14]. This hybrid algorithm has shown to satisfy the QoS requirements of multi-class traffic and allocates bandwidth among traffic classes based on the number of SSs and their respective MRTR for each class. In this hybrid scheme, the EDF is used for SSs of the ertPS, and the rtPS and the WFQ are used for SSs of the nrtPS and the BE classes. The bandwidth has been proven to be allocated in a fair manner, and the overall bandwidth distribution is executed at the beginning of every frame. The EDF and WFQ algorithms are executed at the arrival of every packet. The following equations are used for the calculation of the overall bandwidth allocation.

$$
\begin{gathered}
B_{\text {ertPS,rtPS }}=C \times \frac{\sum_{i \in \text { ertPS,rtPS } \operatorname{MRTR}_{i}}}{\sum_{j=1}^{n} \operatorname{MRTR}_{i}} \\
B_{\text {nrtPS,BE }}=C \times \frac{\sum_{i \in \mathrm{nrtPS}, \mathrm{BE} \mathrm{MRTR}_{i}}}{\sum_{j=1}^{n} \operatorname{MRTR}_{i}},
\end{gathered}
$$

where $C$ is the UL channel capacity, $B_{\text {ertPS,rtPS }}$ is the bandwidth allocated to SS of ertPS and $\operatorname{rtPS}, B_{\mathrm{nrtPS}, \mathrm{BE}}$ is the bandwidth allocated to SS of nrtPS and BE, and MRTR $R_{i}$ is the minimum reserved traffic rate of the SS.

This hybrid algorithm explicitly caters all the required QoS parameters associated to the respective traffic classes in the IEEE 802.16. However, this is not sufficient since scheduling classes have multiple QoS parameters such as rtPS requiring delay, packet loss and throughput guarantee.

The next hybrid scheduling algorithm discussed is the WRR+RR which was proposed by [33]. The WRR and $R R$ algorithms were implemented with a strict priority mechanism for the overall bandwidth allocation. In this hybrid scheme the WRR algorithm has been used to allocate bandwidth among SSs of rtPS and nrtPS classes and the RR algorithm for the SSs of BE class [34]. This hybrid algorithm has shown to starve lower priority SSs in the presence of a large number of higher priority SSs. The algorithm can also result in low fairness among SSs [12].

The hybrid scheme encompassing of EDF+DFPQ scheduling algorithm adds to the collection of hybrid schedulers and was proposed by [12]. This scheme is tailored for four traffic classes. The EDF algorithm is used to serve SSs of the UGS and the rtPS traffic class, the Deficit Fair Priority Queue (DFPQ) algorithm is used to serve the SSs of the nrtPS and the BE traffic class. The proposed algorithm for UL traffic scheduled is based on the current queue in order of the SSs to solve starvation of the lower priority SSs. This is especially in the case of a large number of SSs that comprises of the higher priority. At first the EDF scheduling algorithm is execute for UGS traffic of SSs. Due to the fact that the UGS generates fixed size data packets on a periodic basis and it guarantees the bandwidth for UGS queue, each packet entering in the UGS queues is marked with a deadline. The packet with a smaller deadline will be transmitted earlier. The rtPS traffic follows the same way as the UGS. The rtPS queue will execute after the UGS. The DFPQ scheduling algorithm is used for both nrtPS and BE traffic. The DFPQ algorithm is suitable for the UL traffic located at the SS scheduler. The DFPQ algorithm has similar features as the DRR algorithm which has been deliberated in the literature. This algorithm requires accurate knowledge of the packet size where the packet size vary. In the DFPQ scheduler in each service round, the nrtPS queue is served first until its assigned bandwidth finds deficit, and then the BE service flow queue gets a chance to be served. Therefore, the DFPQ scheduler can guarantee the minimum bandwidth for every non real time services such as nrtPS and BE connection and maintain throughput. This DFPQ scheduling algorithm eliminates the starvation of the lower priority service classes. This hybrid scheme has reduced successfully both the delay for real-time applications and guaranteed the throughput of non-real-time 
applications Table 2 shows hybrid scheduling algorithms with different QoS classes used.

\subsubsection{Constraints of the Hybrid (EDF+WFQ+FIFO) algorithm}

The details of the hybrid algorithm is briefly deliberated prior to engaging in the limitation. The hybrid scheduling algorithm comprising of EDF+WFQ+FIFO was originally proposed by [13]. This algorithm allocates the bandwidth to SSs in a strict priority manner. The EDF scheduling algorithm is used to serve the SSs of type ertPS and rtPS classes, while the WFQ scheduling algorithm is used to serve the SSs of the nrtPS class and FIFO is used to serve SSs of the BE class. The static and strict priorities have been analyzed in detail for their inherent limitations by many researchers. In this section, these identifications have been deliberated with their respective solutions to serve as a basis for the novelty of the proposed algorithm in this research. The original algorithm proposed by [13] does not take into consideration the variable channel conditions of each SS and constraints the flexibility of WiMAX by not providing different bandwidth grant sizes for different quality of service classes. This hybrid algorithm has another drawback which is the starvation of the lower priority SSs (i.e., nrtPS and BE) in the case when large numbers of SSs comprising of higher priority (ertPS and rtPS) are present. This is because the overall available bandwidth is allocated by a strict priority to the SSs of the high priorities [12,35].

\section{Proposed earliest expiry first algorithm}

In the area of scheduling algorithm, the EDF algorithm was originally proposed by [28] and has formed the foundation of many hybrid algorithms. The EDF algorithm has proven to be suitable for real-time traffic for both the ertPS class and the rtPS class where QoS adherence is a must. In analyzing the EDF, the core component of choice is based on a deadline; in specific, it is directed to acquiring the minimum deadline of the packet which is to be served first. The EDF algorithm mechanism guarantees that packets are always searched from the queue accordingly to this minimum value at time ' $t$ ' and is scheduled based on an ascending order. In parallel to the execution of packets in this order, the other packets waiting specially for having different deadlines are kept waiting in the queue to be served until their respective deadline order has arrived. Therefore, in the EDF algorithm, the absolute deadline is pursued with no other mechanism to monitor its compliance. The waiting time of the packets in the queue continuously increases, subsequently increasing the delay. These accumulation of delay may in many instances cause a breach of the stipulated deadline, as no form of monitoring is provided. The adverse effect of this would be packets being consumed by the base station for processing which have actually lost their value due to surpassing the deadlines. This EDF algorithm design has therefore limitations for packets having different deadlines which are confined to waiting extensively in the queue.

\section{Dynamics of the EEF scheduling algorithm}

The proposed earliest expiry first (EEF) algorithm has been designed as an extraction from the hybrid subscheduling EDF algorithm. The proposed EEF algorithm has controls and monitors packets using their deadline and respective expiry. Thus, packets are scheduled based on a combination of stipulated deadlines and the on-going experienced delay. Packets which have longer deadlines are scheduled first as opposed to those with shorter deadlines. These cases only happen when the packets with a shorter deadline has a longer expiry time as compared to the packets with longer deadlines. The proposed EEF algorithm has given a solution to avoid packet waiting time, missed deadline, reducing delay, and increasing better performance of the system. Figure 4 illustrates the proposed EEF algorithm scheduling a packet.

Figure 4 shows that those packets which are waiting in the queue and are more sensitive to expiring their deadlines are served first; the value associated to each packet is time, and each of which relate to the current simulation time, the time the packet arrived into the queue, the period or duration of the time (i.e., waiting time) which is dynamically computed, the deadline associated to each packet, and finally the proposed time to expire. The time to expire is constantly updated over a period of time. The time to expire is computed as the difference between the associated deadline and the waiting time. Thus, unlike the EDF, the proposed EEF has a real-time indicator on the incurred delay. The proposed EEF algorithm uses a

Table 2 Comparative summary of hybrid scheduling algorithms

\begin{tabular}{|c|c|c|}
\hline Author & Hybrid algorithm & QoS classes \\
\hline Wongthavarawat and Ganz [29] & $E D F+W F Q+F I F O$ & $(E D F \Rightarrow r t P S),(W F Q \Rightarrow n r t P S),(F I F O \Rightarrow B E)$ \\
\hline Vinay et al. [30] & $E D F+W F Q$ & $(E D F \Rightarrow r t P S),(W F Q \Rightarrow n r t P S, B E)$ \\
\hline Settembre et al. [33] & $W R R+R R$ & $(W R R \Rightarrow r t P S, n r t P S),(R R \Rightarrow B E)$ \\
\hline Gidlund and Wang [14] & $E D F+W F Q$ & $(E D F \Rightarrow \operatorname{ertPS}, r t P S),(W F Q \Rightarrow n r t P S, B E)$ \\
\hline Chowdhury and Misra [12] & $E D F+D F P Q$ & $(E D F \Rightarrow U G S, r t P S),(D F P Q \Rightarrow n r t P S, B E)$ \\
\hline
\end{tabular}




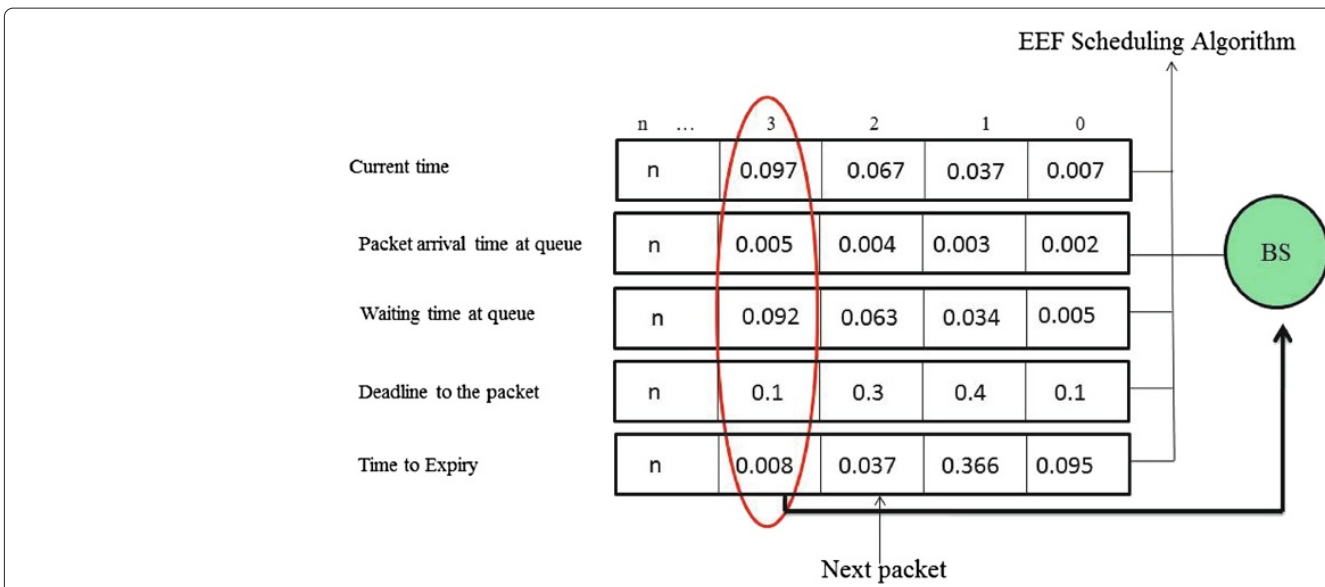

Figure 4 The EEF scheduling algorithm mechanism.

technique which is able to further ensure the deadline compliance. The EDF schedules packets based on the shortest deadline and has no provision for the expiring status of packets which are yet to be served. Therefore, the proposed EEF algorithm proposes to address this by the dual deployment of scheduling algorithm with which the primary element is the stipulated deadline with a secondary element that parallelly exerts expiry monitoring. This can be viewed as a window of caution to ensure packets do not expire by changing the scheduling order. The computation of the expiry is done by first computing the current delay of packets in the queue. This is done by extracting the arrival time of the packets from the current simulation time. The next step involves the computation of the current difference (CDIFF); this parameter is done by 'subtracting' the current delay from the deadline (current delay - deadline). Packets with time to expiry (TTE) values approaching to zero are given priority. A TTE value of zero indicates that the deadline is close to convergence. In the presence of such a case, the packets which have a shorter expiry are given priority even in the case where packets having a shorter deadline are present. This EEF algorithm is shown in Figure 5.

\subsection{Simulation}

The performance of the proposed algorithm EEF has been evaluated by conducting extensive discrete event simulation with each containing a total number of 36 SSs. The main components of the developed WiMAX simulator were oriented on the BS and SSs. The BS embodies the scheduling algorithms. Each sub-scheduling algorithm within the hybrid diameters was individually executed (i.e., ertPS, rtPS, nrtPS and BE). The simulation run time was $50 \mathrm{~s}$ to ensure an equal platform as with the comparative algorithm. In this research, the total number of experiment conducted was six each being oriented by the number of SS. The traffic rate for each class used in the simulation is shown in Table 3. The traffic rate values for the ertPS class is $64 \mathrm{Kbps}$, the rtPS class is $500 \mathrm{Kbps}$, the nrtPS is $500 \mathrm{Kbps}$, and the BE is $64 \mathrm{Kbps}$. The combination of the four traffic classes are represented by the ratio of SSs 3:1:1:1 with 36 SSs and equates to 18 ertPS SSs, 6 rtPS SSs, 6 nrtPS SSs, and 6 BE SSs. This is computed by the summation of the ratio which results in 6 . The ratio of traffic is therefore multiplied by the results of 36 nodes divided by the total ratio which is 6 . Therefore, the total traffic load used is 6,800 Kbps; the ertPS supplied 1,000 Kbps, the rtPS supplied 3,000 Kbps, the nrtPS supplied 2,500 Kbps, and the BE supplied $300 \mathrm{Kbps}$. The service time (i.e., $\mu$ ) equivalent to $20 \mathrm{Mhz}$ is used. The simulation parameters used are shown in Table 3.

\subsection{Performance metrics}

The performance metrics used in this research to analyze the performance of UL scheduling algorithms are throughput, average delay, total missed deadline packet ratio, and average queue utilization. The selection of these metrics is based on the substantial adoption of these parameters for the performance analysis in UL scheduling [13]. These performance metrics and their respective derivation are as follows:

Average throughput $=$ number of packet departures/simulation time

Average delay $=$ total delay/number of packet departures

Average delay $=$ total delay/number of packet departures

misdl ratio $=$ tpkt misdl/npa, 


\begin{tabular}{|c|c|}
\hline & 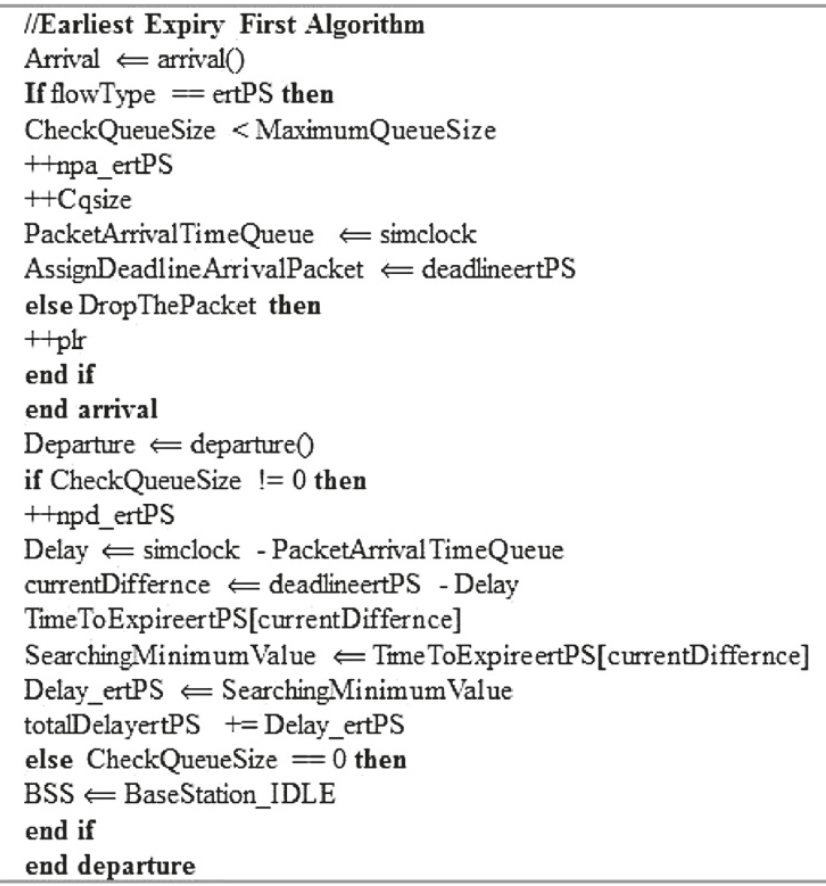 \\
\hline
\end{tabular}

Table 3 Main parameters of the simulation model

\begin{tabular}{|c|c|c|c|c|}
\hline & Values & Packet size & Traffic rate & Traffic load $(\lambda)$ \\
\hline \multicolumn{5}{|l|}{ Input simulation parameter } \\
\hline BS & 1 & & & \\
\hline Number of SSs & 6 to 36 & & & \\
\hline Number of experiments & 6 & & & \\
\hline Ratio of SSS & $3: 1: 1: 1$ & & & \\
\hline Simulation time & $50 \mathrm{~s}$ & & & \\
\hline Service time & $20 \mathrm{Mhz}$ & & & \\
\hline Voice & & 23 bytes & & \\
\hline Video & & 150 to 300 bytes & & \\
\hline FTP & & 150 bytes & & \\
\hline HTTP & & 100 bytes & & \\
\hline ertPS & & & $64 \mathrm{Kbps}$ & \\
\hline rtPS & & & $500 \mathrm{Kbps}$ & \\
\hline nrtPS & & & $500 \mathrm{Kbps}$ & \\
\hline BE & & & $64 \mathrm{Kbps}$ & \\
\hline ertPS & & & & $1,000 \mathrm{Kbps}$ \\
\hline rtPS & & & & $3,000 \mathrm{Kbps}$ \\
\hline nrtPS & & & & 2,500 Kbps \\
\hline $\mathrm{BE}$ & & & & $300 \mathrm{Kbps}$ \\
\hline
\end{tabular}




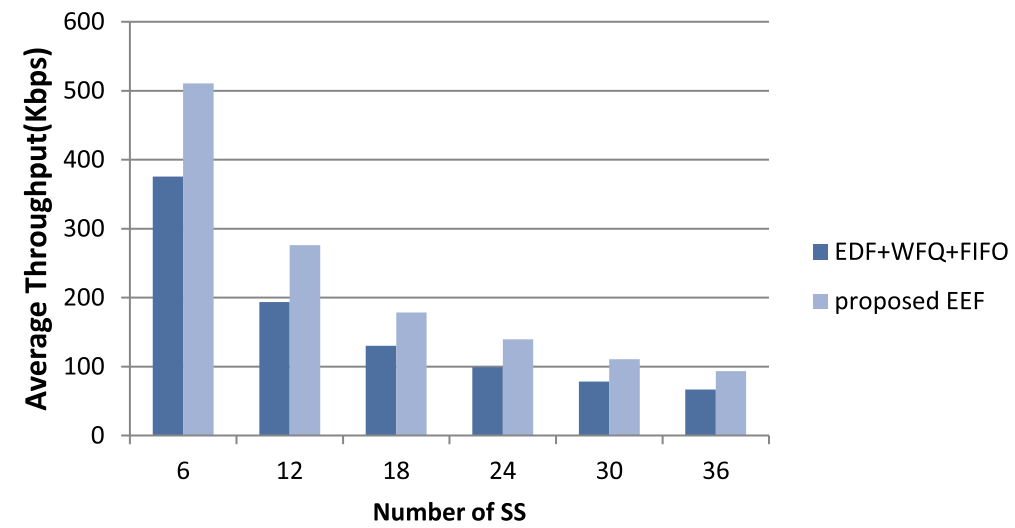

Figure 6 Average throughput versus the number of SS for the ertPS traffic class.

where misdl ratio is the missed deadline ratio, tpkt misdl is the total packets missed deadline, and npa is the number of packets arrived.

$$
\text { avqueue size }=(\text { tqueue size }) /(\text { time }),
$$

where avqueue size is the average queue size, tqueue size is the total queue size, and time is the time $\Leftarrow$ simulation clock.

\section{Results and discussion}

This section presents the performance of the proposed EEF algorithm in comparison with the hybrid EDF+WFQ+FIFO algorithm. The performance metric average throughput per SS is calculated in Kbps, the average delay is calculated in millisecond (ms), the missed deadline packets ratio is calculated in percentage (\%), and the average queue size utilization ratio is also calculated in percentage (\%). The average throughput results for the ertPS class are illustrated in Figure 6.

The results have shown that the average throughput in contrast to the number of SS is considered as the load. This decreases with the increase of the number of
SS. The highest average throughput for the ertPS class is more than $500 \mathrm{Kbps}$ and the lowest is just below $100 \mathrm{Kbps}$. The proposed EEF algorithm has achieved higher throughput as compared to the benchmark hybrid EDF+WFQ+FIFO algorithm. This average throughput has achieved an improved performance due to the expiry index computation and reflection of a versatile and interactive parameter as compared to the static pre-defined deadline.

The average delay for the traffic class ertPS is shown in the Figure 7. A comparison between the results of the benchmark hybrid EDF+WFQ+FIFO algorithm against the proposed EEF algorithm has shown the ability of the proposed EEF algorithm reduced the average delay. This is due to the reason that many packets wait in the queue and eventually expire as opposed to the proposed EEF algorithm which schedule the packets to ensure conformity of the defined deadline. The highest average delay for the proposed EEF algorithm is below $8 \mathrm{~ms}$ whereas the hybrid EDF+WFQ+FIFO algorithm's highest average delay is above $14 \mathrm{~ms}$. Although the proposed EEF algorithm have shown a gradual increase for the delay, the

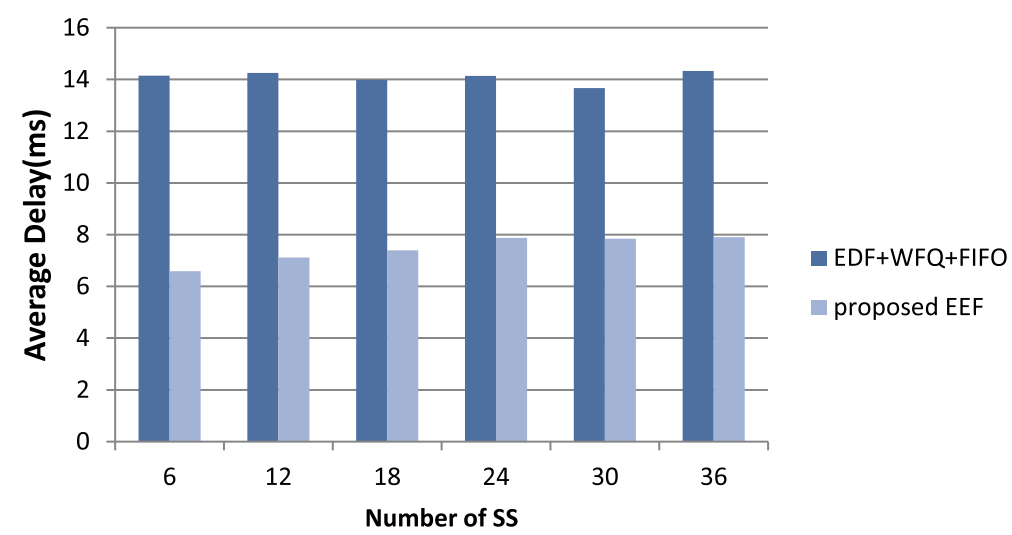

Figure 7 Average delay (ertPS). 


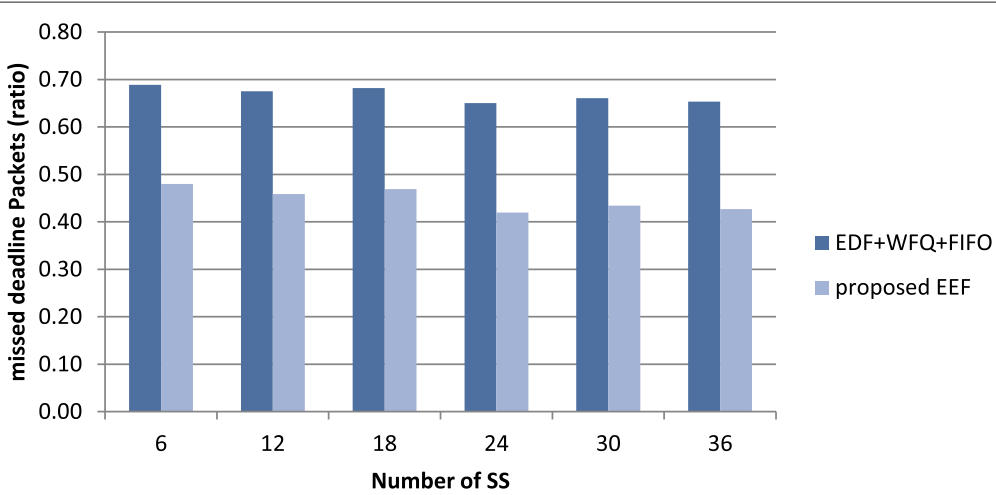

Figure 8 Missed deadline (ertPS).

maximum value achieved before being constantly maintained (i.e., from number of SS equivalent to 24) is still much lower as compared to the hybrid EDF+WFQ+FIFO.

For the class of ertPS, Figure 8 illustrates the packets which have missed their deadline ratio compared to the hybrid EDF+WFQ+FIFO with the proposed EEF algorithm. The proposed EEF algorithm has indeed reduced the ratio of missed deadline packets. This is because of the monitoring of each queued packet that no longer wait indefinitely and is propagated of its priority being served. The maximum range of average dropping of missed deadline packets is and the lowest is above 0.40 . This is in comparison to the base algorithm which has average missed deadline packets below 0.70 .

The Figure 9 illustrates the traffic class of ertPS queue size utilization for the two compared algorithms. The results shows that the hybrid EDF+WFQ+FIFO consumes substantially higher queue space as compared to the proposed EEF algorithm achieves the highest average queue space utilization amounting to a maximum of $23 \%$ and the lowest is $3 \%$. This in contrast for the hybrid benchmark EDF+WFQ+FIFO average queue utilization is has its highest at just below $50 \%$ and the lowest above $8 \%$.
Thus, the proposed EEF has superior performance as compared to the hybrid sub-scheduling EDF algorithm. This is attributed to the uncertain or unlimitless occupancies by packets which eventually breach their deadline. The queue consumption is a function of waiting time. Thus, the proposed EEF mainly improves this factor.

\section{Conclusions}

The IEEE 802.16 WiMAX scheduling and resource management algorithms form the integral parts of this research. The outcome of a detail review of the substantial algorithms within this field has greatly influenced the direction in which the designed and proposed solutions have taken form. This encompassed the identification of the impact hybrid algorithms as the underlying paradigm for serving each distinct class of traffic. In this research, the algorithm tailored at enhancing the collective performance of hybrid algorithms in the WiMAX domains has been designed and developed. The spectrum of constraints which have been extracted from the hybrid EDF+WFQ+FIFO algorithm includes the static nature by which priorities are assigned and maintained during the entire duration of a transmission time. The second constraint is embedded within the EDF scheduling

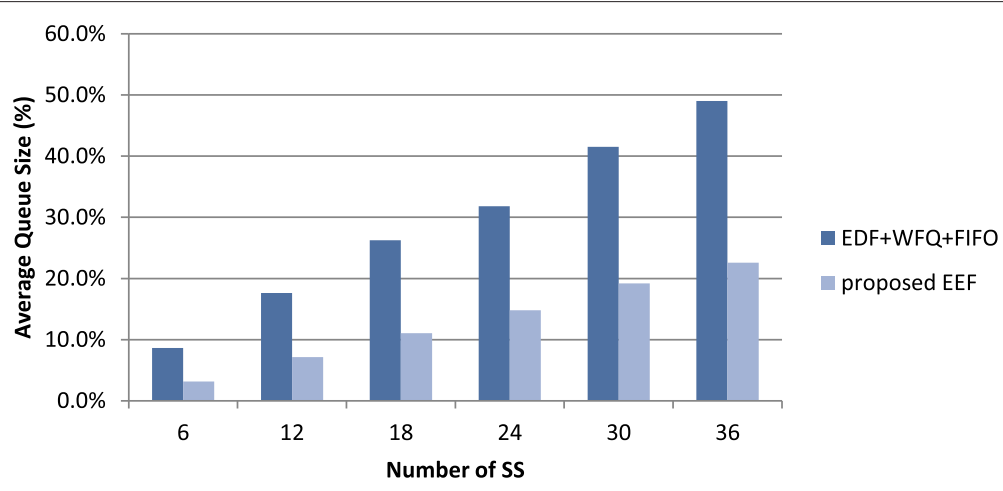

Figure 9 Average queue size (ertPS). 
algorithm and the perseverance of pursuing deadline associated weightages.

The contribution of this research is the reengineering of the scheduling mechanics governing the EDF algorithms. The dominance of the pre-stipulated deadline is indeed acknowledged in the proposed and developed enhanced EEF. However, the significance of providing a monitoring mechanism that gauges between the stipulated and the reality of performance is the central focus of the proposed EEF. The proposed algorithm is able to aptly reevaluate the packets of interest for transmission in the case when packets decline of service due to a longer deadline will eventually breach the deadline tolerance. In such cases, the EEF prioritized with real-time statistics of expiry will serve as the main denominator of priority. The results acquired from the performance analysis conducted have proven the importance of a check-and-balance system by the enhancement achieved across all computed performance metrics.

The future work which would further provide contributions to the elevation of WiMAX includes the incorporation of mobile SS, correlating the different traffic classes in a holistic admission control and the complementary downlink algorithms analysis.

\section{Competing interests}

The authors declare that they have no competing interests.

Received: 24 December 2012 Accepted: 11 September 2014 Published: 23 January 2015

\section{References}

1. GlobalWiMAXsubscriberbasetops20m Telegeography report says. from. http:www.telegeography.com/products/commsupdate/articles//globalwimax-subscriber-base-tops-20m-report-says/. 17/08/2011

2. J Lakkakorpi, A Sayenko, J Moilanen, in Proceedings of the IEEE Wireless Communications and Networking Conference (WCNC 2008), Las Vegas, Nevada, USA. Comparison of different scheduling algorithms for wimax base station: Deficit round-robin vs. proportional fair vs. weighted deficit round-robin, vol. 31, (2008), pp. 1991-1996

3. N El-fishawy, M Zahra, M Ebrahim, M El-gamala, in Radio Science Conference (NRSC), 2011 28th National. Modified cross-layer scheduling for mobile WiMAX networks (IEEE, 2011), pp. 1-10

4. C So-In, R Jain, AK Tamimi, Scheduling in IEEE 802.16e mobile WiMAX networks: key issues and a survey. Selected Areas Commun. IEEE J. 27(2), 156-171 (2009)

5. Z Abichar, Y Peng, J Chang, WiMax: The emergence of wireless broadband. IT Professional. 8(4), 44-48 (2006)

6. J Chen, C Wang, F Tsai, C Chang, S Liu, J Guo, W Lien, J Sum, C Hung, in Proceeding from the 2006 workshop on ns-2: the IP network simulator. The design and implementation of WiMAX module for ns-2 simulator (ACM, 2006), pp. 1-6

7. C Valencia, T Kunz, in Proceedings of the 6th International Wireless Communications and Mobile Computing Conference. Scheduling alternatives for mobile WiMAX end-to-end simulations and analysis (ACM, 2010), pp. 371-375

8. B Li, Y Qin, CP Low, CL Gwee, A survey on mobile WiMAX [Wireless broadband access]. Commun. Mag. IEEE. 45(12), 70-75 (2007)

9. N Rodrigues, WiMAX QoS: Service flow management. Master's thesis, Technical University of Lisbon September (2008)

10. A Kumar, Mobile broadcasting with WiMAX: principles, technology, and applications. (CRC Press, 2008)

11. T-C Tsai, C-H Jiang, C-Y Wang, CAC and packet scheduling using token bucket for IEEE 802.16 networks. J. Commun. 1(2), 30-37 (2006)
12. P Chowdhury, I Misra, A fair and efficient packet scheduling scheme for IEEE 802.16 broadband wireless access systems. arXiv preprint arXiv:1009.6091 (2010)

13. N Ali, P Dhrona, H Hassanein, A performance study of uplink scheduling algorithms in point-to-multipoint WiMAX networks. Comput. Commun. 32(3), 511-521 (2009)

14. M Gidlund, G Wang, Uplink scheduling algorithms for QoS support in broadband wireless access networks. J. Commun. 4(2), 133-142 (2009)

15. L Nuaymi, WiMAX: technology for broadband wireless access. (John Wiley \& Sons, 2007)

16. M Arhaif, Comparative study of scheduling algorithms in WiMAX. Int. J. Sci. Eng. Res. 2(2), 1-7 (2011)

17. M Oktay, H Mantar, in Applied Machine Intelligence and Informatics (SAMI), 2011 IEEE 9th International Symposium on. A real-time scheduling architecture for IEEE 802.16?WiMAX systems (IEEE, 2011), pp. 189-194

18. H Zhang, Service disciplines for guaranteed performance service in packet-switching networks. Proc. IEEE. 83(10), 1374-1396 (1995)

19. A Khalil, A Ksentini, in IWDYN'07 Workshop. Classification of the uplink scheduling algorithms in IEEE 802.16 (Rennes, France, INSA Rennes, 2007)

20. A Sayenko, O Alanen, J Karhula, T Hämäläinen, in Proceedings of the 9th ACM international symposium on Modeling analysis and simulation of wireless and mobile systems. Ensuring the QoS requirements in 802.16 scheduling (ACM, 2006), pp. 1-6

21. J Nagle, On Packet Switches with Infinite Storage. Commun. IEEE Trans 35(4), 435-438 (1987)

22. A Demers, S Keshav, S Shenker, in ACM SIGCOMM Computer Communication Review. Analysis and simulation of a fair queueing algorithm, vol. 19 (ACM, 1989), pp. 1-12

23. A Parekh, R Gallager, A generalized processor sharing approach to flow control in integrated services networks: the single-node case. Netw. IEEE/ACM Trans. 1(3), 344-357 (1993)

24. N Ruangchaijatupon, L Wang, $Y \mathrm{Ji}$, in Communications and Information Technologies, 2006 ISCIT'06. International Symposium on. A Study on the Performance of Scheduling Schemes for Broadband Wireless Access Networks (IEEE, 2006), pp. 1008-1012

25. A Belghith, L Nuaymi, in Wireless Conference, 2008. EW, 2008. 14th European. Comparison of WiMAX scheduling algorithms and proposals for the rtPS QoS class (IEEE, 2008), pp. 1-6

26. M Shreedhar, G Varghese, Efficient fair queuing using deficit round-robin Netwo. IEEE/ACM Trans. 4(3), 375-385 (1996)

27. P Dhrona, A performance study of uplink scheduling algorithms in point to multipoint WiMAX networks Master Thesis, Queen's University Canada (2008)

28. D Ferrari, D Verma, A scheme for real-time channel establishment in wide-area networks. Select. Areas Commun. IEEE J. 8(3), 368-379 (1990)

29. K Wongthavarawat, A Ganz, Packet scheduling for QoS support in IEEE 802.16 broadband wireless access systems. Int. J. Commun. Syst. 16, 81-96 (2003)

30. K Vinay, N Sreenivasulu, D Jayaram, D Das, in Wireless and Optical Communications Networks, 2006 IFIP International Conference on. Performance evaluation of end-to-end delay by hybrid scheduling algorithm for QoS in IEEE 802.16 network (IEEE, 2006), pp. 1-5

31. V Sivaraman, F Chiussi, Statistical analysis of delay bound violations at an earliest deadline first (EDF) scheduler. Perform. Eval. 36, 457-470 (1999)

32. L Georgiadis, R Guerin, A Parekh, Optimal multiplexing on a single link: delay and buffer requirements. Inf. Theory IEEE Trans. 43(5), 1518-1535 (1997)

33. M Settembre, M Puleri, S Garritano, P Testa, R Albanese, M Mancini, V Lo Curto. Computer Networks, 2006 International Symposium on (IEEE, 2006), pp. 11-16

34. T Al-Khasib, H Alnuweiri, H Fattah, V Leung, in Communications, 2005. ICC 2005, 2005 IEEE International Conference on. Mini round robin: an enhanced frame-based scheduling algorithm for multimedia networks, vol. 1 (IEEE, 2005), pp. 363-368

35. W Mardini, M Alfool, Modified WRR scheduling algorithm for WiMAX networks. Netw. Protoc. Algorithms. 3(2), 24-53 (2011)

\section{doi:10.1186/1687-1499-2015-3}

Cite this article as: Oad et al:: Enhanced uplink scheduling algorithm for efficient resource management in IEEE 802.16. EURASIP Journal on Wireles Communications and Networking 2015 2015:3. 